Pacific Journal of Mathematics

LIE HOMOMORPHISMS OF OPERATOR ALGEBRAS 


\title{
LIE HOMOMORPHISMS OF OPERATOR ALGEBRAS
}

\section{ROBERT Miers}

\begin{abstract}
A mapping $\phi: M \rightarrow N$ between *-algebras $M, N$ which is *-linear, and which preserves the Lie bracket $[X, Y]=X Y-$ $Y X$ of elements $X, Y$ in $M$ is called a Lie *homomorphism or just a Lie homomorphism. The main result of this paper states that if $\phi: A \rightarrow B$ is a uniformly continuous Lie *-homomorphism of the $\mathrm{C}^{*}$-algebra $A$ onto the $C^{*}$-algebra $B$ then there exists a central projection $D$ in the weak closure of $B$ such that modulo a center-valued *-linear map which annihilates brackets, $D \phi$ is a *homomorphism and $(I-D) \phi$ is the negative of a *anti-homomorphism.
\end{abstract}

Previously we showed that if $M$ is a factor then so is $N$ and in this case $\phi=\sigma+\lambda$ where $\sigma$ is a ${ }^{*}$-isomorphism or the negative of $a$ *-anti-isomorphism of $M$ onto $N$ and $\lambda$ is a *-linear functional which annihilates brackets in $M$. This result parallels the algebraic theorems of L. Hua and W. S. Martindale.

The main techniques used in this paper are the algebraic techniques of Martindale [8], [9], and Herstein [3]. Adaptations of them allow us to characterize Lie *-isomorphisms between von Neumann algebras and also ultra-weakly $(=U W)$ closed Lie *-ideals which contain the center. For a complete exposition concerning Lie structures on associative algebras we recommend Herstein [4]. We wish to thank Professor H. A. Dye for many invaluable conversations during the preparation of this paper.

1. Preliminaries and notation. We denote by $\mathscr{L}(H)$ the ring of all linear operators $T: H \rightarrow H, H$ a complex Hilbert space with inner product $(.,$.$) , which are bounded in the norm \|T\|=\sup _{\|x\| \leqq 1}\|T x\|$. With this norm, $\mathscr{L}(H)$ is a Banach algebra with identity the identity operator $I$. In addition to the uniform topology on $\mathscr{L}(H)$ we shall be concerned with (1) the weakest topology making the linear functionals $T \rightarrow(T x, y)$ continuous for all $x, y \in H$, called the weak (operator) topology and (2) the weakest topology making the linear functionals $T \rightarrow \sum_{n=1}^{\infty}\left(T x_{n}, y_{n}\right)$ continuous for all sequences $\left\{x_{n}\right\}\left\{y_{n}\right\}$ such that $\sum_{n=1}^{\infty}\left\|x_{n}\right\|^{2}, \sum_{n=1}^{\infty}\left\|y_{n}\right\|^{2}<\infty$ called the ultra-weak (operator) topology. To each operator $T \in \mathscr{L}(H)$ there corresponds an operator $T^{*} \in$ $\mathscr{L}(H)$, called the adjoint of $T$, defined by $(T x, y)=\left(x, T^{*} y\right)$ for all $x, y \in H$. If $T=T^{*}, T$ is called self-adjoint; if $T$ is selfadjoint and $T=T^{2}, T$ is called a projection; if $T T^{*}=T^{*} T=I, T$ is called unitary. A $C^{*}$-algebra, $M$, is a subalgebra of $\mathscr{L}(H)$ which is closed in the 
uniform topology and closed under the operation of taking adjoints. A von Neumann algebra is a weakly closed *-subalgebra of $\mathscr{L}(H)$ which contains $I$. It is a fact that if $M$ is a von Neumann algebra then $M$ is the smallest weakly closed linear subspace of $L(H)$ containing the set $\{P \mid P$ a projection in $M\}$. The set $Z_{M}=\{S \in M \mid S T=$ $T S$ for all $T \in M$ \} is called the center of $M$. If $P$ is a projection in $M$ then $M_{P}=\{P A P \mid A \in M\}$.

Any associative algebra $M$ can be made into a Lie algebra by defining a new multiplication of elements $A, B \in M$ to be the commutator $[A, B]=A B-B A$ where $A B$ is the associative product. For notation let $[M, M]$ be the linear span of all such commutators. A Lie ideal of $M$ is a linear subspace which is an ideal with respect to the multiplication $[A, B]$. Any associative ideal is a Lie ideal, $[M, M]$ is a Lie ideal, and any linear subspace of $Z_{M}$ is a Lie ideal.

We use Dixmier [1], [2] as general references.

\section{General results.}

Definition. Let $M$ be a von Neumann algebra with center $Z_{M}$. For each self-adjoint operator $A \in M$ we define the core of $A$, denoted by $A$, to be the LUB $\left[S \in Z_{M} \mid S\right.$ self adjoint, $\left.S \leqq A\right]$. One has $A-$ $\underline{A} \geqq 0$. Further, if $S \in Z_{M}$ and $A-\underline{A} \geqq S \geqq 0$ then $S=0$. If $P$ is a projection it is clear that $\underline{P}$ is the largest central projection $\leqq P$. We call a projection core-free if $\underline{P}=0$.

The following theorem is a generalization of a result of L. Hua [5] and its proof is due to H. A. Dye.

THEOREM 1. Let $M$ be a von Neumann algebra on the Hilbert space $H$. If $A$ is a self-adjoint operator in $M$ satisfying the identity $[[[X, A], A]=[X, A]$ for all $X \in M$, then $A-\underline{A}$ is a projection.

Proof. The identity is true for $A-\underline{A}$ as well so we can assume $\underline{A}=0$ and $A \geqq 0$. In particular, for each nonzero central projection $C, 0$ lies in the spectrum of $C A$ (considered as an operator on $C H$ ) (otherwise there exists $\varepsilon>0$ such that $A \geqq \varepsilon C$ ).

Suppose $\sigma(A) \neq\{0,1\}$. We can then choose $\mu \in \sigma(A)$ such that $d(\mu,\{0,1\})>2 \varepsilon>0$ where $d(a, S)=$ distance from $a$ to the set $S$. Let $\delta>0$ be such that $\left|t^{3}-t\right|<\delta$ implies $d(t,\{0,1\})<\varepsilon$. By the spectral theorem there exists an operator $B$, commuting with $A$, of the form $B=\sum_{i=1}^{n} \lambda_{i} P_{i}$ where the $\lambda_{i}$ are distinct and $\geqq 0$, the $P_{i}$ are mutually $\perp$ projections of sum $I, 0 \leqq B \leqq A$, and $\|A-B\|$ is so small that $\|A-B\|<\varepsilon$ and

(1) $\|[[[X, B], B], B]-[X, B]\|<\delta$ for all $X$ in $M$ such that $\|X\| \leqq 1$. 
Furthermore, 0 occurs among the $\lambda_{i}$ and $\bar{P}_{i}=I$ (if $P$ is a projection $\bar{P}$ is the central carrier of $P$ ) for $i=1, \cdots, n$. Finally, since $\|A-B\|<\varepsilon$, one of the $\lambda_{i}$, say $\lambda_{2}$, will be at distance $>\varepsilon$ from $\{0,1\}$.

Now we consider the mapping $\psi_{u}(S)=U S U^{-1}$ where $U$ is unitary in $M$. Taking $X=U$ in (1) and rewriting this relation in terms of $\psi_{u}$ we have

$$
\left\|\psi_{u}(B)^{3}-3 B \psi_{u}(B)^{2}+3 B^{2} \psi_{u}(B)-B^{3}-\psi(B)+B\right\|<\delta
$$

$\bar{P}_{i}=I$ implies the existence of nonzero projections $Q_{1} \leqq P_{1}, Q_{2} \leqq P_{2}$ such that $Q_{1} \sim Q_{2}$. We choose a unitary operator $U$ such that $\psi_{u}$ has order $2, \psi_{u}\left(Q_{1}\right)=Q_{2}$ and $\psi_{u}$ is the identity off $Q_{1}+Q_{2}$. For this $U$, (2) reduces to $\left\|\left(\lambda_{2}^{3}-\lambda_{2}\right) Q_{1}+\left(\lambda_{2}^{3}-\lambda_{2}\right) Q_{1}\right\|<\delta$. In particular, $\mid \lambda_{2}^{3}-$ $\lambda_{2} \mid<\sigma$ so that $d\left(\lambda_{2},\{0,1\}\right)<\varepsilon$ which is a contradiction. Hence $\sigma(A) \subseteq$ $\{0,1\}$ and $A$ is a projection.

LeMMA 1. Let $P$ and $Q$ be commuting core-free projections in $M$. Then $P+Q \in Z_{M}$ implies $P \perp Q$, and $P-Q \in Z_{M}$ implies $P=Q$.

Proof. If $P+Q \in Z_{M}$ then $(P+Q)^{2}-(P+Q)=2 P Q \in Z_{M}$. But in this case $P Q \leqq \underline{P}=0$. If $P-Q \in Z_{M}$, then $(P-Q)^{2}+(P-Q)=$ $2(P-P Q) \in Z_{M}$. But $P-P Q \leqq \underline{P}=0$ so that $P=P Q$. By symmetry $Q=P Q$.

Definition. Two projections $P$ and $Q$ are called parallel $(P \| Q)$ if $\bar{P} \bar{Q}=0$.

Lemma 2. $P \| Q$ implies $[[P, X], Q]=0$. Conversely, if $P$ and $Q$ are commuting core-free projections such that $[[P, X], Q]=0$ for all $x \in M$ then $P \| Q$.

Proof. The first statement is clear from the definition of central carrier.

For the other part, multiplying the relation $[[P, X], Q]=0$ on the left by $P Q$ gives $P Q X(I-P)(I-Q)=0$ for all $X \in M$ so that $P Q \|(I-P)(I-Q)$. Multiplying the relation on the left by $P$ and on the right by $Q$ gives $P(I-Q) X Q(I-P)=0$ for all $X \in M$ so that $P(I-Q) \| Q(I-P)$. The first parallelism statement implies $\overline{P Q} \subseteq$ $P \cup Q$. Thus $\overline{P(I-Q) P Q}$ lies in $P \cup Q$, is orthogonal to $Q$, and hence is in $\mathrm{P}$. Since $\underline{P}=0$ this implies $\overline{P(I-Q) P Q}=0$. Likewise $\overline{Q(I-}$ $\overline{P) P Q}=0$, forcing $\overline{P Q}=P Q$ so that $P Q=0$. Thus $P=P(I-Q)$ and $Q=Q(I-P)$ which implies $P \| Q$.

Lemma 3. Let $P$ and $Q$ be commuting projections in $M$ such that 
$[[[[X, P], Q], P], Q]+[[Y, P], Q]=0$ for all $X \in M$. Then there exists a projection $C \in Z_{M}$ such that $P Q(I-C)=0,(I-P)(I-Q) C=0$.

Proof. Multiplying the bracket identity on the left by $P Q$ gives $P Q X(I-P)(I-Q)=0$ for all $X$ so that $P Q \|(I-P)(I-Q)$. Let $C=\overline{P Q}$.

Lemma 4. If the von Neumann algebra $M$ has no summands of type $I_{1}$ ( = central abelian projection) then each nonzero central projection $C \in M$ is the carrier of a core-free projection in $M$.

Proof. Nonzero core-free projections exist in $M$ since if $P$ is a noncentral projection, $\bar{P}-P$ is core-free. By Zorn, let $\left\{P_{\alpha}\right\}$ be a maximal collection of $\|$ core-free projections and let $P=\Sigma P_{\alpha}$. Note that $\bar{P}=I$. For, otherwise there is a central projection $C \neq 0$ orthogonal to all the $P_{\alpha}$, and this $C$ would dominate a nonzero core-free projection thus contradicting maximality. Moreover $P$ is core-free. If $C$ is a central projection and $C \leqq P$, then $\bar{P}_{\alpha} C \leqq P_{\alpha}$ so that $\bar{P}_{\alpha} C=$ 0. Finally $\overline{C P}=C \bar{P}=C$ for any projection $C \in Z_{M}$.

Lemma 5. Let $M$ be any von Neumann algebra, $P$ and $Q$ projections in $M$ with $\bar{P}=\bar{Q} \neq 0$. If $A \in M$ commutes with $P X Q$ and $Q X P$ for all $X \in M$, then $A$ commutes with $P X P$ and $Q X Q$ for all $X \in M$.

Proof. Since $\bar{P} \cdot \bar{Q} \neq 0$ there exist nonzero projections $P_{1} \leqq P$, $Q_{1} \leqq Q$ such that $P_{1} \sim Q_{1}$ 。 Let $V_{1} \in M$ be such that $V_{1}^{*} V_{1}=P_{1}, V_{1} V_{1}^{*}=$ $Q_{1}$. Since $P_{1} X P_{1}=P P_{1} X V_{1}^{*} Q_{1} Q V_{1} P_{1} P$ we have that $A$ commutes with $P_{1} X P_{1}$ for all $X$. If $P_{1} \neq P$, then $\overline{P-P_{1}} \bar{Q} \neq 0$ so there exist projections $P_{2} \leqq P_{1}-P_{1}, Q_{2} \leqq Q$ with $P_{2} \sim Q_{2}$. Let $V_{2} \in M$ be such that $V_{2}^{*} V_{2}=$ $P_{2}, \quad V_{2} V_{2}^{*}=Q_{2}$. As before $P_{2} X P_{2}=P P_{2} X V_{2}^{*} Q_{2} Q V_{2} P_{2} P$ so that $A$ commutes with $P_{2} X P_{2}$ for all $X \in M$. Moreover, since $P_{1} X P_{2}=$ $P P_{1} X V_{2}^{*} Q V_{2} P_{2} P, A$ commutes with $P_{1} X P_{2}$ (and similarly with $P_{2} X P_{1}$ ) for all $X \in M$.

By Zorn, choose a maximal collection $\left\{P_{\alpha}\right\}$ of non-zero mutually orthogonal projections such that (i) $P_{\alpha} \leqq P$, (ii) $A$ commutes with $P_{\alpha} X P_{\beta}$ for all $\alpha, \beta$ and all $X \in M$. By maximality $\Sigma P_{\alpha}=P$. Thus $P X P=\left(\Sigma P_{\alpha}\right) X\left(\Sigma P_{\alpha}\right)$ and so $A$ commutes with $P X P$. A similar statement holds for $Q X Q$.

Lemma 6. Let $M$ be a $C^{*}$-algebra of operators on $H$. If $X, Y \in$ $M$ with $Y$ self-adjoint, and if $[X, Y] \in Z_{M}$ then $[X, Y]=0$.

Proof. By (Singer [13] p. 242), if $X, Y$ skew adjoint, then $[[X$, 
$Y], Y]=0$ implies $[X, Y]=0$. The same statement holds for $X, Y$ self adjoint since then $i X$ and $i Y$ are skew-adjoint.

Now suppose $Y$ self-adjoint. Then $[X, Y] \in Z_{M}$ implies $\left[-X^{*}\right.$, $Y] \in Z_{M}$ and hence $\left[X-X^{*}, Y\right] \in Z_{M}$. By the above $\left[X-X^{*}, Y\right]=0$. Moreover - $\left[-X^{*}, Y\right] \in Z_{M}$ so $\left[X+X^{*}, Y\right] \in Z_{M}$ and is therefore zero. Finally $[X, Y]=0$.

\section{Near-isomorphisms of von Neumann algebras.}

DEFINITION. A mapping $\phi: M \rightarrow N$ between von Neumann algebras $M$ and $N$ which is *-linear and which preserves the lie bracket of operators in $M$ is called a Lie *-homomorphism (or just Lie homomorphism). If $h_{M}(X)=X+Z_{M}$ is the natural Lie homomorphism of $M$ onto $M / Z_{M}$ we call $\phi L$-onto if $h_{N} \circ \phi$ maps $M$ onto $N / Z_{N}$. This implies $\phi\left(Z_{M}\right) \subseteq Z_{N}$. Thus $\phi$ (if $L$-onto) induces a Lie homomorphism of $M / Z_{M}$ onto $N / Z_{N}$. If this induced homomorphism is a Lie isomorphism call $\phi$ a near-isomorphism.

If $\phi: M \rightarrow N$ is a $L$-onto Lie homomorphism, and $P \in M$ then by Theorem 1 and Lemma 1 there exists a unique core-free projection $\theta(P) \in N$ and a central element $\lambda(P)(=\phi(P))$ such that $\phi(P)=\theta(P)+$ $\lambda(P)$. If we write $\theta^{\prime}(P)=\overline{\theta(P)}-\theta(P)$, then $\theta^{\prime}(P)$ is core-free, $\phi(P)$ $=-\theta^{\prime}(P)+\lambda^{\prime}(P)+\lambda^{\prime}(P)\left(\lambda^{\prime}(P) \in Z_{N}\right)$ and this representation is unique. Note that $\overline{\theta(P)}=\overline{\theta^{\prime}(P)}$ and that $P \in Z_{M}$ implies $\theta(P)=0$. We assume from now on that $\phi$ is a near-isomorphism between the von Neumann algebras $M$ and $N$.

Lemma 7. If $Q$ is a core-free projection in $N$, then there exists a core-free projection $P \in M$ such that $\theta(P)=Q$.

Proof. $[[[Y, Q] Q] Q]-[Y, Q]=0$ for all $Y \in N$. Let $P^{\prime} \in M$ be such that $\phi\left(P^{\prime}\right)-Q \in Z_{N}$. Then $\phi\left(\left[\left[\left[X, P^{\prime}\right] P^{\prime}\right] P^{\prime}\right]-\left[X, P^{\prime}\right]\right)=0$ for all $X \in M$ so that $\left[\left[\left[X, P^{\prime}\right] P^{\prime}\right] P^{\prime}\right]-\left[X, P^{\prime}\right] \in$ ker $\phi \leqq Z_{M}$. By Lemma 6 this bracket expression is zero. Hence, by Theorem $1, P^{\prime}-\underline{P}^{\prime}=P$ a core-free projection. $\phi\left(P^{\prime}\right)=\theta(P)+\lambda(P)+\phi\left(\underline{P}^{\prime}\right)=Q+Z, z \in Z_{M}$ and $\lambda(P)+\phi\left(\underline{P}^{\prime}\right) \in Z_{N}$. Hence by Lemma $1, \theta(P)=Q$.

LeMma 8. Let $P$ and $Q$ be core-free projections in $M$. Then $P \| Q$ if and only if $\theta(P) \| \theta(Q)$ and $\bar{P}=\bar{Q}$ if and only if $\overline{\theta(P)}=\overline{\theta(\bar{Q})}$.

Proof. $P \| Q$ implies $[[P, X], Q]=0$ for all $X \in M$. If $Y \in N$ let $X \in M$ be such that $\dot{\phi}(X)-Y \in Z_{N}$. Then $\phi([[P, X], Q)=[[\theta(P), Y]$, $\theta(Q)]=0$. Hence $\theta(P) \| \theta(Q)$. On the other hand if $\theta(P) \| \theta(Q)$ then $[[P, X], Q] \in \operatorname{ker} \dot{\phi} \subseteq Z_{M}$ for all $X \in M$. By Lemma $6,[[P, X] Q]=0$ 
for all $X$.

For the other part, if $\bar{P}=\bar{Q}$ but $\overline{\theta(P)} \neq \overline{\theta(Q)}$ then there exists a projection $C \in Z_{N}$ such that $C \theta(Q)=0, C \theta(P) \neq 0$. There exists a corefree projection $R$ in $M$ such that $\theta(R)=C \theta(P)$. Hence $\theta(R) \| \theta(Q)$ which implies $R \| Q$. But $\theta(R) \| \theta(P)$ so that $R \| P$ a contradiction. Similarly $\overline{\theta(\bar{P})}=\overline{\theta(Q)}$ implies $\bar{Q}=\bar{P}$.

LEMMA 9. $\theta$ and $\theta^{\prime}$ are additive on parallel core-free projections.

Proof. Let $P_{1}, \cdots, P_{n}$ be parallel core-free projections in $M$. By Lemma 8 the $\theta\left(P_{1}\right), \cdots, \theta\left(P_{n}\right)$ are parallel (and core-free) so that $\theta\left(P_{1}\right)+\cdots+\theta\left(P_{n}\right)$ is a projection. It is also core-free by parallelism. One has

$$
\theta\left(P_{1}+\cdots+P_{n}\right)-\sum_{i=1}^{n} \theta\left(P_{i}\right)=\sum_{i=1}^{n} \lambda\left(P_{i}\right)-\lambda\left(P_{1}+\cdots+P_{n}\right) \in Z_{N}
$$

By Lemma 1

$$
\theta\left(\sum_{i=1}^{n} P_{i}\right)=\sum_{i=1}^{n} \theta\left(\mathrm{P}_{i}\right)\left(\text { and } \sum_{i=1}^{n} \lambda\left(P_{i}\right)=\lambda\left(\sum_{i=1}^{n} P_{i}\right)\right) .
$$

A similar argument gives the result for $\theta^{\prime}$.

Lemma 10. If $M$ and $N$ have no central summands of type $I_{1}$ then there exists a unique ${ }^{*}$-isomorphism \& of $Z_{M}$ onto $Z_{N}$ such that $\theta(C P)=\psi(C) \theta(P)$ for all projections $P$ and all central projections $C$. One has $\psi(\bar{P})=\overline{\theta(P})$. A similar statement holds with $\theta$ replaced by $\theta^{\prime}$.

Proof. We first define $\psi$ for central projections. For each central projection $C$, choose a core-free projection $P$ such that $\bar{P}=C$ (Lemma 4). Define $\psi(C)=\overline{\theta(P})$. If $Q$ is any other core-free projection such that $\bar{Q}=C$ then $\overline{\theta(Q)}=\overline{\theta(P)}$ by Lemma 8 so that the mapping is well defined. If $D$ is a central projection in $N$, choose a core-free projection $R \in N$ such that $\bar{R}=D$. There exists a core-free projection $P \in$ $N$ such that $\theta(P)=R$ so that $\psi(\bar{P})=\overline{\theta(P)}=\bar{R}=D$. Hence $\psi$ is onto. If $\psi(\bar{P})=\psi(\bar{Q})$ for core-free projections $P$ and $Q$ then $\overline{\theta(P)}=\theta(Q)$ and by Lemma $8, \bar{P}=\bar{Q}$. If $C$ and $D$ are central projections in $M$ with $C D=0$ let $P$ and $Q$ be core-free projections in $M$ with $\bar{P}=C, \bar{Q}=D$. Then $C D=0$ iff $\bar{P} \bar{Q}=0$ iff $\bar{P} \| \bar{Q}$ iff $\theta(P) \| \theta(Q)$ iff $\overline{\theta(P) \theta(Q)}=0$ iff $\psi(C) \psi(D)=0$. Thus $\psi$ is a projection orthoisomorphism of $Z_{M}$ on $Z_{N}$ and as such is implemented by a unique *-isomorphism (also called $\psi$ ) of $Z_{M}$ on $Z_{N}$.

To show $\theta(C P)=\psi(C) \theta(P)$ choose a core-free projection $Q$ such that $\bar{Q}=C(I-\bar{P})$. Then $P C+Q$ has carrier $C$, and $\psi(C)=\overline{\theta(P C+Q)}$ $=\overline{\theta(P C})+\overline{\theta(Q)}$ by Lemma 9. Now $\theta(P)=\theta(P C)+\theta(P(I-C))$ and 
both terms on the right are orthogonal to $\overline{\theta(Q)}$. Multiplying the two relations together we have $\psi(C) \theta(P)=\theta(C P)$.

Finally we show that $\theta(C P)=\psi(C) \theta(P)$ implies $\psi(\bar{P})=\overline{\theta(\bar{P})}$. Put $C=\bar{P}$ to get $\theta(P)=\psi(\bar{P}) \theta(P)$ or $\theta(P) \leqq \psi(\bar{P})$. So $\overline{\theta(P}) \leqq \psi(\bar{P})$. Now if $\bar{P}+\bar{R}=I$ and $\bar{P} \perp \bar{R}$ we have $\overline{\theta(\bar{P})}+\overline{\theta(R)}=1$.

Definition. Two projections $P, Q$ are called co-orthogonal (co $\perp$ ) if $\bar{P}-P \perp \bar{Q}-Q$.

LemMA 11. Let $P_{1}, \cdots, P_{n}$ be commuting core-free projections, each pair of which satisfy the identity of Lemma 3. Then there exists a central projection $C$ such that the $P_{i}$ are orthogonal on $C$, co-orthogonal on $I-C$.

Proof. For each pair $P_{i}, P_{j}(i \neq j)$ there exists a central projection $C_{i j}$ such that $P_{i} P_{j} C_{i j}=0$ and $\left(I-P_{i}\right)\left(I-P_{j}\right)\left(I-C_{i j}\right)=0$. Let $\mathscr{B}$ be the boolean algebra generated by the $C_{i j}$. If $C$ is an atom in $\mathscr{B}$ then $P_{i} P_{j} C=0$ or $\left(I-P_{i}\right)\left(I-P_{j}\right) C=0(i \neq j)$.

Index so that $P_{1} C, \cdots, P_{m} C$ are the non-zero terms of the form $P_{i} C$ (we can leave 0 out since it is both $\perp$ and co $\perp$ to all projections). We claim that these projections are either $\perp$ or co $\perp$. It suffices to take $m \geqq 3$. If $P_{1} P_{2} C=0$ then all the $P_{i} C$ are mutually $\perp$. For if, say, $P_{1} P_{3} C \neq 0$ then $\left(I-P_{1}\right)\left(I-P_{3}\right) C=0$ so that $\left(I-P_{1}\right) C \leqq P_{3}$. The two relations give $P_{2} C \leqq P_{3}$. Now if $C P_{2} P_{3}=0$ we have $P_{2} C=$ 0 a contradiction. If $\left(I-P_{2}\right)\left(I-P_{3}\right) C=0$ then $C\left(I-P_{2}\right) \leqq P_{3}$ so that $C \leqq P_{3}$ contradicting the fact that $\underline{P}_{3}=0$. The same argument shows $P_{1} P_{i} C=0$ for $i \geqq 3$. Applying this reasoning to each $P_{i} C$ in turn gives their mutual perpendicularity.

In a similar way if, say, $\left(I-P_{1}\right)\left(I-P_{2}\right) C=0$ then all the $(I-$ $\left.P_{i}\right) C$ are mutually orthogonal. If, for example, $P_{1} P_{3} C=0$ then $(I-$ $\left.P_{1}\right) C \leqq P_{2}$ and we have $P_{3} C \leqq P_{2} C P_{2} P_{3}=0$ implies $P_{3}=0$ and $\left(I-P_{2}\right) \times$ $\left(I-P_{3}\right) C=0$ implies $C \leqq P_{2}$, both contradictions.

CoRollary. Let $M$ and $N$ be von Neumann algebra with no central summands of type $I_{1}$, and let $P_{1}, \cdots, P_{n}$ be a collection of mutually $\perp$ projections in $M$. There exists a projection $D \in Z_{M}$ such that the $P_{j} D$ have $\perp$ image under $\theta$, the $P_{i}(I-D)$ have $\perp$ image under $\theta^{\prime}$.

Proof. Apply the above to the $\theta\left(P_{i}\right)$ and get the projection $C \in$ $Z_{N}$. One has $C=\psi(D)$ for some $D \in Z_{M}$ and $\theta\left(D P_{i}\right)=C \theta\left(P_{i}\right), \theta\left(D P_{i}\right)=$ $C \theta^{\prime}\left(P_{i}\right)$. 
Lemma 12. Let $P_{1}, \cdots, P_{n}$ be projections in $M$ with $\bar{P}_{i_{0}}=I, D \in$ $Z_{M}$ such that (i) $P_{i} D$ are mutually $\perp$, (ii) $\Sigma P_{i} D \in Z_{M}$, then $\sum_{i=1}^{n} P_{i} D=D$.

Proof. Say $\Sigma P_{i} D=C \in Z_{M}$. Then $P_{i_{0}} D=P_{i_{0}} C D$ so that $D=C D$. Hence $D \leqq C$. Obviously $C \leqq D$.

Lemma 13. Let $P_{1}, \cdots, P_{n}$ and $D$ as in Corollary to Lemma 11 with $\quad \bar{P}_{i_{0}}=I . \quad$ Then $\psi(D) \sum_{i=1}^{n} \theta\left(P_{i}\right)=\psi(D), \quad \psi(I-D) \sum_{i=1}^{n} \theta\left(P_{i}\right)=$ $\psi(I-D)$.

Proof. $\quad \phi(D)=\sum_{i=1}^{n} \theta\left(P_{i} D\right)+\sum_{i=1}^{n} \lambda\left(P_{i} D\right) \in Z_{N}$. Hence $\sum_{i=1}^{n}$ $\theta\left(P_{i}\right) \psi(D) \in Z_{N}$ and the $\theta\left(P_{i}\right) \psi(D)$ are mutually $\perp$. By Lemma 12, $\sum_{i=1}^{n} \theta\left(P_{i}\right) \psi(D)=\psi(D)$. A similar argument works for $\theta^{\prime}$ and $I-D$.

LEMma 14. If $M$ is any von Neumann algebra and $P$ a core-free projection in $M$, then $M_{P}(=\{P A P \mid A \in M\}) \cap Z_{M}=\{0\}$.

Proof. Suppose $P A P \in Z_{M}$ where $A$ is self-adjoint and $A \leqq I$. Then $((P-P A P) x, x)=((I-A) P x, P x) \geqq 0$ so that $P \geqq P A P$. Since $\underline{P}=0$ we have $P A P=0$. In general, if $A=A_{1}+i A_{2}$ where $A_{1}, A_{2}$ are (nonzero) self-adjoint and $P A P \in Z_{M}$ then $P A P+P A^{*} P=2 P A_{1} P \in Z_{M}$. Therefore $P\left(1 /\left\|A_{1}\right\|\right) A_{1} P \in Z_{M}$ so that $P A_{1} P=0$ by the first part of the argument. Similarly $P A_{2} P=0$.

4. The decomposition theorem. The following arguments are, in part, adaptations of those of Martindale [8], [9]. These adaptations are of sufficient technical complexity to merit their entire conclusion.

We consider first the case where $\phi: M \rightarrow N$ is a near isomorphism of $M$ to $N$, and $M$ is a type $I_{2}$ von Neumann algebra. Let $P_{1}, P_{2}$ be equivalent, orthogonal, abelian projections of sum $I$ in $M$. We have that $\underline{P}_{i}=0, \bar{P}_{i}=I$ for $i=1,2$, and that $\theta\left(P_{1}\right) \theta\left(P_{2}\right)=0$ since $\theta\left(P_{1}\right)+$ $\theta\left(P_{2}\right) \in Z_{N}$. Moreover, $I=\psi(I)=\psi\left(\bar{P}_{1}\right)=\overline{\theta\left(P_{1}\right)} \leqq \theta\left(P_{1}\right)+\theta\left(P_{2}\right)=\theta\left(P_{1}\right)+$ $\theta\left(P_{2}\right) \leqq I$ and so $\theta\left(P_{1}\right)+\theta\left(P_{2}\right)=I$. Notice that $\theta\left(P_{1}\right)=\theta^{\prime}\left(P_{2}\right)$ and $\theta\left(P_{2}\right)=$ $\theta^{\prime}\left(P_{1}\right)$. For notation let $M_{i j}=\left\{P_{i} A P_{j} \mid A \in M\right\}, N_{i j}=\left\{\theta\left(P_{i}\right) A \theta\left(P_{j}\right) \mid A \in N\right\}$.

LEMMA 15. $\phi^{-1}\left(N_{11}+N_{22}\right)=M_{11}+M_{22}$.

Proof. If $X \in M_{11}$ let $Y=\phi(X)$. Since $\left[X, P_{2}\right]=0$ we have $0=$ $\left[Y, \theta\left(P_{2}\right)\right]$ or $Y \theta\left(P_{2}\right)=\theta\left(P_{2}\right) Y$. This implies $\theta\left(P_{2}\right) Y \theta\left(P_{1}\right)=\theta\left(P_{1}\right) Y \theta\left(P_{2}\right)=$ 0 which in turn implies $Y \in N_{11}+N_{22}$. Similarly if $X \in M_{22}$.

Suppose $Y \in N_{11}$ and $X \in M$ is such that $\phi(X)-Y \in Z_{N}$. Then $\left[Y, \theta\left(P_{2}\right)\right]=0$ so that $\left[X, P_{2}\right] \in \operatorname{ker} \phi \cong Z_{M}$ which implies $\left[X, P_{2}\right]=0$ 
by Lemma 6 .

Lemma 16. $\phi^{-1}\left(N_{i j}\right)=M_{i j}(i \neq j)$.

Proof. For example, suppose $i=1, j=2$ and let $X \in M_{12} . \quad X=$ $\left[Q_{1},\left[X, Q_{2}\right]\right]=\left[Q_{1}, X\right]$. Hence $\phi(X)=\left[\theta\left(P_{1}\right),\left[\phi(X), \theta\left(P_{2}\right)\right]\right]=\theta\left(P_{1}\right) \phi(X)$ $\theta\left(P_{2}\right)+\theta\left(P_{2}\right) \phi(X) \theta\left(P_{1}\right)=\theta\left(P_{1}\right)\left[\theta\left(P_{1}\right), \phi(X)\right] \theta\left(P_{2}\right)+\theta\left(P_{2}\right)\left[\theta\left(P_{1}\right), \phi(X)\right] \theta\left(P_{1}\right)=$ $\theta\left(P_{1}\right) \phi(X) \theta\left(P_{2}\right)-\theta\left(P_{2}\right) \phi(X) \theta\left(P_{1}\right)$ since $\theta\left(P_{1}\right) \perp \theta\left(P_{2}\right)$. Therefore $\phi(X)=$ $\theta\left(P_{1}\right) \phi(X) \theta\left(P_{2}\right)$. So $\phi\left(M_{12}\right) \subseteq N_{12}$.

If $Y \in N_{12}$, then $Y=\left[\theta\left(P_{1}\right), Y\right]=\left[\phi\left(P_{1}\right), Y\right]$ and also $Y=\left[\theta\left(P_{1}\right)\right.$, $\left.\left[Y, \theta\left(P_{2}\right)\right]\right]=\left[\phi\left(P_{1}\right),\left[Y, \phi\left(P_{2}\right)\right]\right]$. Let $X \in M$ be such that $\phi(X)-Y \in$ $Z_{N}$. Then $\left[P_{1}, X\right]-\left[P_{1},\left[X, P_{1}\right]\right] \in \operatorname{ker} \phi$ and so equals zero, or $P_{1} X-$ $X P_{1}=P_{1} X P_{2}+P_{2} X P_{1}$. This gives $P_{2} X P_{1}=0$. Now $\phi(X)=\phi\left(P_{1} X P_{1}\right)+$ $\phi\left(P_{1} X P_{2}\right)+\phi\left(P_{2} X P_{2}\right)$ so that $\phi\left(P_{1} X P_{1}\right)+\phi\left(P_{1} X P_{2}\right)+\phi\left(P_{2} X P_{2}\right)-Z=Y$ for some $Z \in Z_{N}$. By the first part of the argument we then have $\phi\left(P_{1} X P_{1}\right)+\phi\left(P_{2} X P_{2}\right)-Z \in N_{12}$. By Lemma 15, $\phi\left(P_{1} X P_{1}\right)+\phi\left(P_{2} X P_{2}\right)=$ $\theta\left(P_{1}\right) S \theta\left(P_{1}\right)+\theta\left(P_{2}\right) T \theta\left(P_{2}\right)$. Hence $\theta\left(P_{1}\right) S \theta\left(P_{1}\right)+\theta\left(P_{2}\right) T \theta\left(P_{2}\right)-Z=$ $\theta\left(P_{1}\right) W \theta\left(P_{2}\right)$ for some $W \in N$. Multiplying on the left and right by $\theta\left(P_{1}\right)$ gives $\theta\left(P_{1}\right) S \theta\left(P_{1}\right)-Z \theta\left(P_{1}\right)=0$. Similarly $\theta\left(P_{2}\right) T \theta\left(P_{2}\right)-Z \theta\left(P_{2}\right)=$ 0 . Hence $\theta\left(P_{1}\right) S \theta\left(P_{1}\right)+\theta\left(P_{2}\right) T \theta\left(P_{2}\right)-Z=0$. This implies $\phi\left(P_{1} X P_{2}\right)=$ $Y$.

Corollary. $\phi$ is onto.

LEMMA 17. $\phi\left(M_{i i}\right) \subseteq N_{i i}+Z_{N}$.

Proof. $M_{11}$ and $M_{22}$ are abelian von Neumann algebras in their own right and so, therefore are $M_{11}+M_{22}\left(=M_{11} \oplus M_{22}\right)$ and by Lemma 15, $N_{11}+N_{22}\left(=N_{11} \oplus N_{22}\right)$. The latter implies that $N_{11}, N_{22}$ are abelian.

Let $X \in M_{11}$ and $Y=\phi(X)$. Then $Y \in N_{11}+N_{22} \subseteq Z_{N_{11}}+Z_{N_{22}}$, (since $N_{11}+N_{22}$ abelian $)=Z_{N 11}+Z_{N 22}=Z_{\theta\left(P_{1}\right)}+Z_{\theta\left(P_{2}\right)}$. Thus $Y=S \theta\left(P_{1}\right)+$ $T \theta\left(P_{2}\right)$ where $S, T \in Z_{N}$. But $\theta\left(P_{1}\right)=I-\theta\left(P_{2}\right)$ so that $Y=(S-$ T) $\theta\left(P_{1}\right)+T$.

We define mappings $\sigma: M \rightarrow N$ and $\lambda: M \rightarrow Z_{N}$ in the following manner: if $A \in M_{i j}(i \neq j)$ then $\sigma(A)=\phi(A)$, and if $A \in M_{i i}$ by Lemma 17, $\phi(A)=\sigma(A)+Z$ where $\sigma(A) \in N_{i i}$ and $Z \in Z_{N}$. Extend $\sigma$ to all of $M$ by linearity and define $\lambda(A)=\phi(A)-\sigma(A) . \quad \sigma$ and $\lambda$ are well defined, for if $A_{1}+Z_{1}=A_{2}+Z_{2}$ where $A_{i} \in N_{i i}, Z_{i} \in Z_{N}$ then $A_{1}-A_{2} \in N_{i i} \cap$ $Z_{N}=\{0\}$.

LEMMA 18. $\sigma$ and $\lambda$ are linear mappings.

Proof. We show $\sigma(\alpha A+\beta B)=\alpha \sigma(A)+\beta \sigma(B)$ for $A, B \in M$ and 
$\alpha, \beta \in \mathrm{C}$. It suffices to assume $A, B \in M_{i i}$. Then $\sigma(\alpha A+\beta B)-$ $\alpha \sigma(A)-\beta \sigma(B)=-\lambda(\alpha A+\beta B)+\alpha \lambda(A)+\beta \lambda(B) \in N_{i i} \cap Z_{N}=\{0\}$.

LEMMA 19. $\sigma$ and $\lambda$ preserve adjoints.

Proof. Again assume $A \in M_{i i}$. Then $\sigma\left(A^{*}\right)-\sigma(A)^{*}=\lambda(A)^{*}-$ $\lambda\left(A^{*}\right) \in N_{i i} \cap Z_{N}=\{0\}$.

Lemma 20. If $A \in M_{i i}, B \in M_{i j}(i \neq j)$ then $\sigma(A B)=\sigma(A) \sigma(B)$.

Proof. In this case, $\sigma(A B)=\phi(A B)=\phi(A B-B A)=[\phi(A), \phi(B)]=$ $[\sigma(A), \sigma(B)]=\sigma(A) \sigma(B)$.

Lemma 21. If $A, B \in M_{i j}$ then $\sigma(A B)=\sigma(A) \sigma(B)$.

Proof. Let $S \in M_{i j}(i \neq j)$. Then $\sigma(A B) \sigma(S)=\sigma(A B S)=\sigma(A) \sigma(B S)$ $=\sigma(A) \sigma(B) \sigma(S)$. Hence, $\quad[\sigma(A B)-\sigma(A) \sigma(B)] \sigma(S)=\sigma(S) \sigma(A B)-$ $[\sigma(A) \sigma(B)]=0$. Specifically, $\sigma(A B)-\sigma(A) \sigma(B)$ commutes with $N_{i j}$. Similarly $\sigma(A B)-\sigma(A) \sigma(B)$ commutes with $N_{j i}$. Applying Lemma 5, $\sigma(A B)-\sigma(A) \sigma(B)$ commutes with $N_{i i}$ and $N_{j j}$, so that, $\sigma(A B)-$ $\sigma(A) \sigma(B) \in Z_{N} \cap N_{i i}=\{0\}$.

Lemma 22. If $A \in M_{i j}, B \in M_{j i}(i \neq j)$ then $\sigma(A B)=\sigma(A) \sigma(B)$.

Proof. Applying $\phi$ to the identity $[[A, B], A]=2 A B A$ we have $\phi(A B A)=\phi(A) \phi(B) \phi(A)$. But since $\phi=\sigma$ on $M_{i j}(i \neq j)$ we have $\sigma(A B A)=\sigma(A) \sigma(B) \sigma(A) . \quad$ Moreover, $\quad \phi[A, B]=[\phi(A), \phi(B)]=[\sigma(A)$, $\sigma(B)]$ and $\phi[A, B]=\phi(A B)-\phi(B A)=\sigma(A B)+\lambda(A B)-\sigma(B A)-\lambda(B A)$ so that (1) $\sigma(A) \sigma(B)-\sigma(A B)+\sigma(B A)-\sigma(B) \sigma(A)=C \in Z_{N}$. Multiplying this last relation on the right by $\sigma(A)$ we get $C \sigma(A)=$ $\sigma(A) \sigma(B) \sigma(A)-\sigma(A B) \sigma(A)=\sigma(A) \sigma(B) \sigma(A)-\sigma(A B A)=0 . \quad$ Similarly $C \sigma(B)=0$. Multiplying (1) by $C$, and using the preceding, we have (2) $C(\sigma(B A)-\sigma(A B))=C^{2}$. Hence $C^{3}=C^{2}(\sigma(B A)-\sigma(A B))=C(\sigma(B A)-$ $\sigma(A B))^{2}=C(\sigma(B A) \sigma(B A)-\sigma(A B) \sigma(A B))$. Multiplying (1) by $\theta(P)$ gives $C \theta(P)=\sigma(A) \sigma(B)-\sigma(A B)$, and by $I-\theta(P)$ gives $C \theta(1-P)=\sigma(B A)-$ $\sigma(B) \sigma(A) . \quad$ Hence $C^{3}=C\left((\sigma(B) \sigma(A)+C(I-\theta(P)))^{2}-(\sigma(A) \sigma(B)-\right.$ $\left.C \theta(P))^{2}\right)=C\left(\sigma(B) \sigma(A) \sigma(B) \sigma(A)+C^{2}(I-\theta(P))-(\sigma(A) \sigma(B) \sigma(A) \sigma(B)+\right.$ $\left.\left.C^{2} \theta(P)\right)\right)=C^{3}(1-\theta(P))-C^{3} \theta(P)=C^{3}-2 C^{3} \theta(P)$. Thus $C^{3} \theta(P)=0$. In particular the set $N C^{2} \theta(P) N=\left\{X C^{2} \theta(P) Y \mid X, Y \in N\right\}$ is a nilpotent ideal in $M$ so that $C^{2} \theta(P)=0$. By the same reasoning $C \theta(P)=0$.

Lemma 23. $\sigma$ is $a^{*}$-isomorphism of $M$ onto $N$. 
Proof. (i) 1-1: If $\sigma(A)=0$ then $\phi(A B)=\sigma(A B)+\lambda(A B)=$ $\lambda(A B) \in Z_{N}$ for all $B \in M$. Thus, by the near one-one-ness of $\phi, A B \in$ $Z_{M}$ for all $B \in M$. Thus $A, A P_{1}$, and $A P_{2}$ all are in $Z_{M}$. This implies $A P_{i} \in Z_{M} \cap M_{i i}(i=1,2)$ so that $A=A P_{1}+A P_{2}=0$.

(ii) onto: $\sigma=\dot{\phi}$ on $M_{i j}(i \neq j)$ and $\dot{\phi}^{-1}\left(N_{i j}\right)=M_{i j}$. Let $Y \in N_{11}$. There exists $X \in M_{11}+M_{22}$ such that $\phi(X)=Y$. Hence $X=S P_{1}+$ $T P_{2}=(S-T) P_{1}+T$ where $S, T \in Z_{M}$. Thus $Y=\phi\left(P_{1}(S-T) P_{1}+T\right)$ $=\sigma\left(P_{1}(S-T) P_{1}\right)+\lambda\left(P_{1}(S-T) P_{1}\right)+\phi(T)$ so that $Y-\sigma\left(P_{1}(S-T) P_{1}\right) \epsilon$ $N_{11} \cap Z_{N}=\{0\}$.

THEOREM 2. Let $\phi: M \rightarrow N$ be a near-isomorphism of the type $I_{2}$ von Neumann algebra $M$ onto the von Neumann algebra $N$. Then (i) $\phi$ is onto, and (ii) $\dot{\phi}=\sigma+\lambda$ where $\sigma$ is $a^{*}$-isomorphism of $M$ onto $N$ and $\lambda$ is $a^{*}$-linear mapping of $M$ into $Z_{N}$ which annihilates brackets. $N$ is then of type $I_{2}$ with $\theta\left(P_{1}\right), \theta\left(P_{2}\right)$ equivalent, orthogonal, abelian projections of sum $I$.

In what follows we assume $M$ and $N$ have no summands of type $I_{1}$. We now turn our attention to the case when $M$ is not of type $I_{2}$ but has a summand of type $I_{2}$. Choose non-zero orthogonal projections $P_{1}, P_{2}, P_{3}$ such that $\sum_{i=1}^{3} P_{i}=I, \bar{P}_{1}=\bar{P}_{2}=I, I-\bar{P}_{3}$ is the $I_{2}$ summand, $I-\bar{P}_{3} \leqq P_{1}+P_{2}$, and $P_{1}-P_{1} \bar{P}_{3}, P_{2}-P_{2} \bar{P}_{3}$ are the equivalent abelian projections comprising $I-\bar{P}_{3}$. There exists a central projection $D$ such that $\theta\left(P_{i} D\right)$ are mutually $\perp$, and $\theta^{\prime}\left(P_{i}(I-D)\right)$ are mutually $\perp$. For notation, let $Q_{i}=P_{i} D, R_{i}=P_{i}(I-D), T_{i}=Q_{i} \bar{P}_{3}, T_{i}^{\prime}=R_{i} \bar{P}_{3}$ all for $i=1,2,3$, and let $S_{i}=Q_{i}\left(I-\bar{P}_{3}\right), S_{i}^{\prime}=R_{i}\left(I-\bar{P}_{3}\right)$ for $i=1,2$. Furthermore let $M_{i j}=Q_{i} M Q_{j}, M_{i j}=R_{i} M R_{j}, N_{i j}=\theta\left(Q_{i}\right) M\left(Q_{j}\right), N_{i j}=\theta^{\prime}\left(R_{i}\right) N \theta^{\prime}\left(R_{j}\right)$.

Lemma 24. $S_{1}, S_{2}$ are equivalent abelian projections. A similar statement is true for $S_{1}^{\prime}, S_{2}^{\prime}$.

Proof. $\quad P_{1}\left(1-\bar{P}_{3}\right) \sim P_{2}\left(1-\bar{P}_{3}\right)$ and are abelian. Hence $S_{1}=D P_{1}(1-$ $\left.\bar{P}_{3}\right) \sim D P_{2}\left(I-\bar{P}_{3}\right)=S_{2}$. Moreover $S_{1} M S_{1} \subseteq P_{1}\left(1-\bar{P}_{3}\right) M P_{1}\left(1-\bar{P}_{3}\right)$ which is abelian.

Lemma 25. The $S_{i}, S_{i}^{\prime}, T_{j}, T_{j}^{\prime} i=1,2, j=1,2,3$ are mutually $\perp$, core-free projections. A similar statement is true for $\theta\left(S_{i}\right), \theta^{\prime}\left(S_{i}^{\prime}\right)$, $\left.\theta_{(} T_{j}\right), \theta^{\prime}\left(T_{j}^{\prime}\right)$. Moreover

$$
\begin{aligned}
\sum_{i=1}^{3} T_{i} & =D \bar{P}_{3}, \sum_{i=1}^{3} T_{i}^{\prime}=(I-D) \bar{P}_{3}, \sum_{i=1}^{2} \theta\left(S_{i}\right)+\sum_{i=1}^{3} \theta\left(T_{i}\right) \\
& =\psi(D), \sum_{i=1}^{2} \theta^{\prime}\left(S_{i}\right)+\sum_{i=1}^{3} \theta^{\prime}\left(T_{i}\right)=\psi(I-D) .
\end{aligned}
$$

Finally $\left.\overline{\theta\left(S_{1}\right)}=\overline{\theta\left(S_{2}\right)}, \overline{\theta\left(T_{i}\right)}=\overline{\theta\left(T_{j}\right)}, \overline{\theta^{\prime}\left(S_{1}^{\prime}\right)}=\overline{\theta^{\prime}\left(S_{2}^{\prime}\right)}, \overline{\theta^{\prime}\left(T_{i}\right.}\right)=\overline{\theta^{\prime}\left(T_{j}^{\prime}\right)}$. 
Proof. $\theta\left(S_{1}\right)=\theta\left(Q_{1}\right) \psi\left(I-\bar{P}_{3}\right), \quad \theta\left(S_{2}\right)=\theta\left(Q_{2}\right) \psi\left(I-\bar{P}_{3}\right) \quad \sum_{i=1}^{3} \theta\left(T_{i}\right)=$ $\sum_{i=1}^{3} \psi\left(\bar{P}_{3}\right) \theta\left(Q_{i}\right)=\psi\left(\bar{P}_{3}\right) \sum_{i=1}^{3} \theta\left(Q_{i}\right)$. Hence $\theta\left(S_{1}\right)+\theta\left(S_{2}\right)+\sum_{i=1}^{3} \theta\left(T_{i}\right)=$ $\theta\left(Q_{1}\right)+\theta\left(Q_{2}\right)+\psi\left(\bar{P}_{3}\right) \theta\left(Q_{3}\right)=\theta\left(Q_{1}\right)+\theta\left(Q_{2}\right)+\theta\left(Q_{3} \bar{P}_{3}\right)=\sum_{i=1}^{3} \theta\left(Q_{i}\right)=\psi(D)$ by Lemma 13. $\bar{S}_{1}=\overline{P_{1} D}\left(I-\bar{P}_{3}\right)=D\left(I-\bar{P}_{3}\right)=\overline{P_{2} D}\left(1-\bar{P}_{3}\right)=\bar{S}_{2}$. Hence by Lemma $8, \overline{\theta\left(S_{1}\right)}=\overline{\theta\left(S_{2}\right)}$.

LEMMA 26. $\phi^{-1}\left(\sum_{i=1}^{3} N_{i i}+\sum_{i=1}^{3} N_{i i}\right)=\sum_{i=1}^{3} M_{i i}+\sum_{i=1}^{3} M_{i i}$.

Proof. If $X \in M_{11}$ then $0=\left[X, Q_{2}\right]=\left[X, Q_{3}\right]=\left[X, R_{i}\right] i=1,2,3$. Hence, $0=\left[\phi(X), \theta\left(Q_{2}\right)\right]=\left[\phi(X), \theta\left(Q_{3}\right)\right]=\left[\phi(X), \theta^{\prime}\left(R_{i}\right)\right] i=1,2,3$. Thus, $0=\theta\left(Q_{1}\right) \phi(X) \theta\left(Q_{2}\right)=\theta\left(Q_{2}\right) \phi(X) \theta\left(Q_{1}\right)=\theta\left(Q_{3}\right) \phi(X) \theta\left(Q_{2}\right)=\theta\left(Q_{2}\right) \dot{\phi}(X) \theta\left(Q_{3}\right)$, and $\theta^{\prime}\left(R_{i}\right) \phi(X) \theta^{\prime}\left(R_{j}\right)=0 i \neq j$.

On the other hand for $Y \in N_{11}$, then $0=\left[Y, \theta\left(Q_{2}\right)\right]=\left[Y, \theta\left(Q_{3}\right)\right]=$ $\left[Y, \theta^{\prime}\left(R_{i}\right)\right] i=1,2,3$. Thus if $X \in M$ is such that $\phi(X)-Y \in Z_{N}$ we have $0=\left[X, Q_{2}\right]=\left[X, Q_{3}\right]=\left[X, R_{i}\right] \quad i=1,2,3$ by Lemma 6 . Hence $X \in \sum_{i=1}^{3} M_{i i}+\sum_{i=1}^{3} M_{i i}$.

LeMma 27. $\phi^{-1}\left(N_{i j}\right)=M_{i j}$ and $\phi^{-1}\left(N_{j i}\right)=M_{i j}$ for $i \neq j$.

Proof. $\phi\left(M_{i j}\right) \subseteq N_{i j}(i \neq j)$ as in the $I_{2}$ case. Suppose $Y \in N_{12}$ and let $X$ be such that $\phi(X)-Y \in Z_{N} . \quad Y=\left[\theta\left(Q_{1}\right), Y\right]=\left[\theta\left(Q_{1}\right),[Y\right.$, $\left.\left.\theta\left(Q_{2}\right)\right]\right]$. Hence, as before $\left[Q_{1}, X\right]=\left[Q_{1},\left[X, Q_{2}\right]\right]$ so that $Q_{2} \times Q_{1}=0$. Moreover, $0=\left[Y, \theta\left(Q_{3}\right)\right]=\left[Y, \theta^{\prime}\left(R_{i}\right)\right]$ for $i=1,2,3$ so that $0=[X$, $\left.Q_{3}\right]=\left[X, R_{i}\right]$ for $i=1,2,3$ by Lemma 6 . Writing $X=\sum_{1 \leqq i, j \leqq 3} X_{i j}+$ $\sum_{1 \leq i, j \leq 3} \underline{X}_{i j}$ where $X_{i j} \in M_{i j}, \underline{X}_{i j} \in \underline{M}_{i j}$ we have $X=\sum_{i=1}^{3} X_{i i}+\sum_{i=1}^{3} X_{i i}+$ $X_{12}$. Hence $\phi(X)=\sum_{i=1}^{3} X_{i i}+\sum_{i=1}^{3} \underline{X}_{i i}+X_{12}-Z=Y \in N_{12}$ for some $Z \in$ $Z_{N}$. By Lemma 26, $\phi\left(\sum_{i=1}^{3} X_{i i}+\sum_{i=1}^{3} \underline{X}_{i i}\right) \in \sum_{i=1}^{3} N_{i i}+\sum_{i=1}^{3} \underline{N}_{i i}$ and so, as in Lemma 16, $\phi\left(\sum_{i=1}^{3} X_{i i}+\sum_{i=1}^{3} \underline{X}_{i i}\right)=Z$ or $\phi\left(X_{12}\right)=Y$.

For the other part, let $X \in \underline{M}_{12}$. As before, $X=\left[R_{1},\left[X, R_{2}\right]\right]=$ $\left[R_{1}, X\right]$. But now, since $\phi\left(R_{i}\right)=-\theta^{\prime}\left(R_{i}\right)+\lambda^{\prime}\left(R_{i}\right)$ and the $\theta^{\prime}\left(R_{i}\right)$ are mutually $\perp$, we have $\phi(X)=\left[-\theta^{\prime}\left(R_{1}\right),\left[\phi(X),-\theta\left(R_{2}\right)\right]\right]=\theta^{\prime}\left(R_{2}\right) \times$ $\phi(X) \theta^{\prime}\left(R_{1}\right)+\theta^{\prime}\left(R_{1}\right) \phi(X) \theta^{\prime}\left(R_{2}\right)=\theta^{\prime}\left(R_{2}\right) \phi(X) \theta^{\prime}\left(R_{1}\right)-\theta^{\prime}\left(R_{1}\right) \phi(X) \theta^{\prime}\left(R_{2}\right)$. Hence $\phi(X)=\theta^{\prime}\left(R_{2}\right) \phi(X) \theta^{\prime}\left(R_{1}\right)$. This shows $\phi\left(\underline{M}_{i j}\right) \subseteq N_{j i}$. An argument similar to that above shows $\phi^{-1}\left(\underline{N}_{j i}\right)=\underline{M}_{i j}$.

Corollary. $\phi$ is onto.

LEMMA 28. $\phi\left(Z_{M_{11}}\right) \subseteq N_{11}+Z_{N}$.

Proof. $A \in Z_{M_{11}}$ implies $[A, X]=0$ for all $X$ in $M_{23}+M_{32}+$ $\sum_{i=1}^{3} M_{i i}+\sum_{i=1}^{3} \underline{M}_{i i}$. Hence if $B=\phi(A),[B, X]=0$ for all $X$ in $N_{23}+$ $N_{32}+\sum_{i=1}^{3} N_{i i}+\sum_{i=1}^{3} \underline{N}_{i i}$ by Lemma 26 and Lemma 27 . That is, [ $B$, $X]=0$ for all $X$ in $N_{0}=\left\{S T S \mid T \in N, S=\theta\left(Q_{2}\right)+\theta\left(Q_{3}\right)+\psi(I-D)\right\}$. Now by Lemma $26, B=B_{1}+C$ where $B_{1} \in N_{11}, C \in N_{0}$. Thus, $0=$ 
$[B, X]=\left[B_{1}+C, X\right]=[C, X]$ for all $X \in N_{0}$, and $C \in Z_{N_{0}}=\{S Z S \mid Z \in$ $\left.Z_{N}\right\}$. Thus, since $S=I-\theta\left(P_{1}\right), B=B_{1}+C=B_{1}+Z\left(I-\theta\left(P_{1}\right)\right)=$ $B_{1}-Z \theta\left(P_{1}\right)+Z$ where $Z \in Z_{N}$.

CoRollary. $\phi\left(S_{1} M S_{1}\right) \subseteq N_{11}+Z_{N}$.

Proof. $S_{1} M S_{1}$ is an abelian algebra, and so $S_{1} M S_{1} \subseteq Z_{S_{1} M S_{1}}=$ $\left\{Z S_{1} \mid Z \in Z_{N}\right\} \subseteq Z_{M_{11}}$ since $S_{1}=P_{1} D\left(I-\bar{P}_{3}\right)$.

LEMMA 29. $\phi\left(T_{1} M T_{1}\right) \leqq \sum_{i=1}^{2} N_{\theta\left(S_{i}\right)}+\sum_{i=1}^{2} N_{\theta^{\prime}\left(S_{i^{\prime}}\right)}+\sum_{i=1}^{3} N_{\theta\left(T_{i}\right)}+$ $\sum_{i=1}^{3} N_{\theta^{\prime}\left(T_{i}^{\prime}\right)}$.

Proof. Since $I=\sum_{i=1}^{2} \theta\left(S_{i}\right)+\sum_{i=1}^{2} \theta^{\prime}\left(S_{i}^{\prime}\right)+\sum_{i=1}^{3} \theta\left(T_{i}\right)+\sum_{i=1}^{3} \theta^{\prime}\left(T_{i}^{\prime}\right)$ we apply the method of Lemma 15 .

Lemma 30. $\phi\left(T_{i} M T_{j}\right)=\theta\left(T_{i}\right) N \theta\left(T_{j}\right), \phi\left(S_{i} M S_{j}\right)=\theta\left(S_{i}\right) N \theta\left(S_{j}\right)$, $\phi\left(S_{i}^{\prime} M S_{j}^{\prime}\right)=\theta^{\prime}\left(S_{j}^{\prime}\right) N \theta^{\prime}\left(S_{i}^{\prime}\right), \phi\left(T_{i}^{\prime} M T_{j}^{\prime}\right)=\theta^{\prime}\left(T_{j}^{\prime}\right) N \theta^{\prime}\left(T_{i}^{\prime}\right)$ for $i \neq j$.

Proof. As before.

LEMma 31. $\phi\left(T_{1} M T_{1}\right) \subseteq \theta\left(T_{1}\right) N \theta\left(T_{1}\right)+Z_{N}$.

Proof. If $A \in T_{1} M T_{1}$ then $[A, X]+0$ for all $X$ in $T_{2} M T_{3}+T_{3} M T_{2}+$ $\sum_{i \neq j} S_{i} M S_{j}+\sum_{i \neq j} S_{i}^{\prime} M S_{j}^{\prime}+\sum_{i=j} T_{i}^{\prime} M T_{j}^{\prime}$. Hence, if $B=\phi(A),[B, X]$ $=0$ for all $X$ in $\theta\left(T_{2}\right) N \theta\left(T_{3}\right)+\theta\left(T_{3}\right) N \theta\left(T_{2}\right)+\sum_{i \neq j} \theta\left(S_{i}\right) N \theta\left(S_{j}\right)+$ $\sum_{i \neq j} \theta^{\prime}\left(S_{i}^{\prime}\right) N \theta^{\prime}\left(S_{j}^{\prime}\right)+\sum_{i \neq j} \theta^{\prime}\left(T_{i}^{\prime}\right) N \theta^{\prime}\left(T_{j}^{\prime}\right)$. Since the corresponding projections (e.g., $\theta\left(S_{i}\right), \theta\left(S_{j}\right)$ ) have the same central carrier, we can apply Lemma 5 to conclude that $[B, X]=0$ for all $X$ in $\{S T S \mid T \in N, S=$ $\left.\theta\left(T_{2}\right)+\theta\left(T_{3}\right)+\sum_{i=1}^{n} \theta\left(S_{i}\right)+\sum_{i=1}^{2} \theta^{\prime}\left(S_{i}^{\prime}\right)+\sum_{i=1}^{3} \theta^{\prime}\left(T_{i}^{\prime}\right)\right\}=N_{0}$. As before we have $B=B_{1}+C$ where $B_{1} \in \theta\left(T_{1}\right) N \theta\left(T_{1}\right), C \in Z_{N_{0}}$. Hence $B \in$ $\theta\left(T_{1}\right) N \theta\left(T_{1}\right)+Z_{N}$.

LEMMA 32. $\phi\left(M_{11}\right) \subseteq N_{11}+Z_{N}$.

Proof. $M_{11}=P_{1} M P_{1}=\left(S_{1}+T_{1}\right) M\left(S_{1}+T_{1}\right)=S_{1} M S_{1}+T_{1} M T_{1}$. Similar arguments show that $\phi\left(M_{i i}\right) \subseteq N_{i i}+Z_{N}, \dot{\phi}\left(\underline{M}_{i i}\right) \subseteq \underline{N}_{i i}+Z_{N}$ for $i=1,2,3$.

We define mappings $\sigma: M_{D} \rightarrow N_{\psi(D)}$ and $\lambda: M_{D} \rightarrow Z_{N}$ in the following manner: if $A \in M_{i j}(i \neq j)$ then $\sigma(A)=\phi(A)$, and if $A \in M_{i i}$ by Lemma $30 \phi(A)=\sigma(A)+Z$ where $\sigma(A) \in N_{i i}$ and $Z \in Z_{N}$. Extend $\sigma$ to all of $M_{D}$ by linearity and define $\lambda(A)=\phi(A)-\sigma(A)$ for $A \in M_{D}$. $\sigma$ and $\lambda$ are well defined, for if $T_{1}+Z_{1}=T_{2}+Z_{2}$ where $T_{i} \in N_{i i}, Z_{i} \in Z_{N}$, then $T_{1}-T_{2} \in N_{i i} \cap Z_{n}=\{0\}$. 
We can analogusly define mappings $\sigma^{\prime}: M_{I-D} \rightarrow N_{\psi(I-D)}$ and $\lambda^{\prime}$ : $M_{I-D} \rightarrow Z_{N}$ using the corresponding facts for $\underline{M}_{i j}$. Note that $\sigma^{\prime}\left(\underline{M}_{i j}\right)=$ $\underline{N}_{j i}$.

As before, $\sigma, \lambda, \sigma^{\prime}$, and $\lambda^{\prime}$ are *-linear mappings.

Lemma 33. If $A \in M_{i i}, B \in M_{i j}(i \neq j)$ then $\sigma(A B)=\sigma(A) \sigma(B)$. If $A \in \underline{M}_{i i}, B \in \underline{M}_{i j}(i \neq j)$ then $\sigma^{\prime}(A B)=-\sigma^{\prime}(B) \sigma^{\prime}(A)$.

Proof. $A \in \underline{M}_{i i}, B \in \underline{M}_{i j}$ then $\sigma^{\prime}(A B)=\phi(A B)=\phi[A, B]=[\phi(A)$, $\phi(B)]=\left[\sigma^{\prime}(A), \sigma^{\prime}(B)\right]=-\sigma^{\prime}(B) \sigma^{\prime}(A)$ since $\sigma(B) \in \underline{M}_{j i}$.

Lemma 34. If $A, B \in M_{i i}$ then $\sigma(A B)=\sigma(A) \sigma(B)$. If $A, B \in \underline{M}_{i i}$ then $\sigma^{\prime}(A B)=-\sigma^{\prime}(B) \sigma^{\prime}(A)$.

Proof. The proof for $A, B \in M_{i i}$ is similar to Lemma 21. If $A$, $B \in \underline{M}_{i i}$ and $S \in \underline{M}_{i j}(i \neq j)$ then $\sigma^{\prime}(S) \sigma^{\prime}(B) \sigma^{\prime}(A)=-\sigma^{\prime}(B S) \sigma(A)=\sigma^{\prime}(A B S)$ $=-\sigma^{\prime}(S) \sigma^{\prime}(A B)$. Hence $0=\left[\sigma^{\prime}(A B)+\sigma^{\prime}(B) \sigma^{\prime}(A)\right] \sigma^{\prime}(S)=\sigma^{\prime}(S)\left[\sigma^{\prime}(A B)+\right.$ $\left.\sigma^{\prime}(B) \sigma^{\prime}(A)\right]$.

Lemma 35. If $A \in M_{i j}, B \in M_{j i}(i \neq j)$ then $\sigma(A B)=\sigma(A) \sigma(B)$. If $A \in \underline{M}_{i j}, B \in \underline{M}_{j i}$ then $\sigma^{\prime}(A B)=\sigma^{\prime}(B) \sigma^{\prime}(A)$.

Proof. Similar to Lemma 22.

TheOREM 3. $\sigma\left(\operatorname{resp} . \sigma^{\prime}\right)$ is $a^{*}$-isomorphism of $M_{D}$ onto $N_{\psi(D)}$ (resp. is the negative of a *anti-isomorphism of $M_{I-D}$ onto $\left.N_{\psi(I-D)}\right)$ and $\lambda\left(\right.$ resp. $\left.\lambda^{\prime}\right)$ is $a^{*}$ linear map from $M_{D}$ into $Z_{N}$ (resp. from $M_{I-D}$ into $\left.Z_{N}\right)$ which annihilates brackets.

Proof. Similar to the $I_{2}$ case.

REMARK 1. If $M$ has no summand of type $I_{2}$, then a proof much like the one above gives the same Theorem 3 for such an $M$. Thus, including the $I_{2}$ case, Theorem 3 holds for all von Neumann algebras $M$ which have no abelian summands of type $I$.

REMARK 2. A result of Sunouchi [15] (see remarks following Theorem 3 below) shows, in part, that if $M$ is an infinite von Neumann algebra, then $[M, M]=M$. Thus in the above, if $\phi: M \rightarrow N$ is a nearisomorphism and $M$ infinite then $\phi=\sigma+\sigma^{\prime}$ and is automatically bounded. 
REMARK 3. Martindale [10] proved that if $L$ is a Lie derivation of a primitive ring $R$ into itself, where $R$ has a nonzero idempotent and is not of characteristic 2 , then $L=D+T$ where $D$ is an ordinary derivation and $T$ a center-valued additive map which annihilates commutators. By slightly altering Martindale's proof we can show the same result if $R$ is a von Neumann algebra.

4. Lie *-homomorphisms of $\mathrm{C}^{*}$-algebras. We now turn our attention to characterizing uniformly continuous Lie *-homomorphisms between $C^{*}$-algebras. In order to do this we first investigate ultra weakly (UW) closed Lie *-ideals in von Neumann algebras.

Definition. A Lie *-ideal $U$ in a ${ }^{*}$-algebra $M$ is a ${ }^{*}$-linear subspace of $M$ such that if $X \in U$ and $Y \in M$ then $[X, Y] \in U$.

Lemma 36. Let $M$ be a $C^{*}$-algebra, $U$ a Lie *-ideal in $M$ such that if $A, B \in U$ then $[A, B]=0$. Then $U \subseteq Z_{M}$.

Proof. Since $U$ is closed with respect to the *-operation it is generated, as a linear space, by its self-adjoint elements. Suppose $A=$ $A^{*} \in U$, and $B$ any self-adjoint element in $M$. Then $[B, A] \in U$ so that $[[B, A], A]=0$ since $U$ commutative. By Lemma $6,[B, A]=0$.

Lemma 37. If $U$ is a Lie *-ideal in a $C^{*}$-algebra $M$ which is at the same time an associative subring of $M$ then either $U \subseteq Z_{M}$ or $U$ contains $a$ (two-sided, associative) ideal of $M$.

Proof. We follow Herstein [3: Theorem 2, p. 281]. If $U$ is commutative then $U \subseteq Z_{M}$ by Lemma 36. If $U$ is not commutative then there exist $X, Y \in U$ such that $[X, Y] \neq 0$. In this case Herstein proves that the ideal $M[X, Y] M$ (= all finite linear combinations of elements of the form $S[X, Y] T$ where $S, T \in M)$ is contained in $U$.

LEMma 38. If $U$ is any Lie ideal in an associative ring $M$, then $T(U)=\{T \in M \mid[T, X] \in U$ for all $X \in M\}$ is a Lie ideal and subring of $M$. Moreover $U \subseteq T(U)$. If $U$ is a Lie *ideal and ultra-weakly closed, so is $T(U)$.

Proof. See Herstein [3: Theorem 2, p. 282].

CoRollary. If $M$ is a von Neumann algebra, $U$ a ultra-weakly closed Lie ${ }^{*}$-ideal in $M$, then $T(U)=Z_{M}$ or there exists a nonzero 
central projection $C$ in $M$ such that $M_{C} \subseteq T(U)$.

Proof. By Lemma 37 either $T(U) \subseteq Z_{M}$ (in which case $T(U)=$ $Z_{M}$ since $Z_{M} \subseteq T(U)$ ), or there exists a nonzero two-sided ideal $I \subseteq$ $T(U)$. In the latter case, $I^{-U W} \leqq T(U)$ and $I^{-U W}=M_{C}$ for some nonzero central projection $C$.

THEOREM 4. If $M$ is a von Neumann algebra and $U$ an ultraweakly closed Lie *-ideal in $M$, then $T(U)=Z_{M}$ or there exists a nonzero central projection $C$ such that $T(U)=Z_{M}+M_{C}$.

Proof. By the corollary to Lemma 38, $T(U)=Z_{M}$ or there exists a nonzero central projection $C^{\prime}$ such that $M_{C}^{\prime} \subseteq(U)$. Let $C$ be a maximal such projection. Then $M_{C} \subseteq T(U)$ so that $Z_{M}+M_{C} \subseteq T(U)$.

Suppose $S \in T(U)$ and $S \notin Z_{M}+M_{C}$. We can assume $S=S^{*}$. Writing $S=S C+S(I-C)$ we see that $S(I-C) \in T(U)$ (since $S, S C \in$ $T(U)$ ) and that $S(I-C) \neq 0$ (since $S \notin Z_{M}+M_{C}$ ). Since $S(I-C) \notin$ $Z_{M}$ there exists $S_{1}=S_{1}^{*} \in M$ such that $\left[S_{1}, S(I-C)\right] \neq 0$. Moreover $\left[S_{1}, S(I-C)\right] \in T(U)$ since $T(U)$ is a Lie ideal, and $\left[\left[S_{1}, S(I-C)\right]\right.$, $S(I-C)] \neq 0$ by Lemma 6. Applying the techniques of Herstein [3: Theorem 2, p. 281] with $X=\left[S_{1}, S(I-C)\right], Y=S(I-C)$, the set $M[X, Y] M$ is a non-zero two sided ideal contained in $T(U)$. Or, $0 \neq$ $(I-C) M\left[\left[S_{1}, S\right], S\right] M \subseteq U$. Let $J=M\left[\left[S_{1}, S\right], S\right] M$. Then $((I-$ $C) J)^{-U W}=(I-C) J^{-U W}=(I-C) M_{D}$, where $D$ is a nonzero central projection, is a nonzero, two-sided, ultraweakly closed ideal in $T(U)$. Hence $(I-C) M_{D}+M_{C}$ is an ultraweakly closed two-sided ideal in $T(U)$ properly containing $M_{C}$, a contradiction. Thus $T(U)=Z_{M}+M_{C}$.

Sunouchi [14] proved that if $M$ is a von Neumann algebra, and $[M, M]=$ all finite linear combinations of brackets from $M$, then $M$ infinite implies $[M, M]=M, M$ finite implies that $[M, M]$ is uniformly dense in the null space of the center-valued trace, \#, on $M$. Using this fact and Theorem 3 we can prove the following corollary:

CoROLlary. If $U$ is an ultra-weakly closed Lie*-ideal in a von Neumann algebra $M$, and if $Z_{M} \subseteq U$, then either $U=Z_{M}$ or there exists a nonzero central projection $C$, in $M$, such that $U=Z_{M}+M_{C}$.

Proof. If $T(U)=Z_{M}$ then $Z_{M} \subseteq U \subseteq T(U) \subseteq Z_{M}$. Otherwise $T(U)=Z_{M}+M_{C}$ for some non-zero central projection $C \in M$. From the definition of $T(U)$ this implies $C[M, M]=\left[M_{C}, M_{C}\right] \subseteq U$.

Case 1. $M$ infinite. The $[M, M]=M$ so that $C[M, M]=M_{C} \leqq U$. Thus $Z_{M}+M_{C} \subseteq U \subseteq T(U) \subseteq Z_{M}+M_{C}$. 
Case 2. $M$ finite. Then $[M, M]^{-\|} \|=\left\{X \in M \mid X^{\sharp}=0\right\}$ where \# is the center-valued trace on $M$. For notation let $N_{C}=\left\{X C \mid(X C)^{\sharp}=0\right\}$. We have $\left[M_{C}, M_{C}\right]^{-11} \|=N_{C}$ so that $N_{C} \subseteq U$. This implies that $Z_{M}+$ $N_{C} \subseteq U \subseteq T(U) \subseteq Z_{M}+M_{C}$. But for arbitrary $X \in M, X C=(X C)^{\sharp}+$ $X C-(X C)^{\sharp} \in Z_{M}+N_{C} \subseteq U$. Thus $M_{C} \subseteq U$.

THEOREM 5. If $\phi: M \rightarrow N$ is an UW-continuous L-onto Lie *-homomorphism between the Neumann algebras $M$ and $N$ then there exists a central projection $C \in M$ and another $U W$-continuous $L$-onto Lie *-homomorphism \& from $M$ to $N$ such that

(1) the difference of $\psi$ and $\phi$ is a $\lambda$-map (i.e., *-linear from $M$ to $Z_{N}$, annihilates brackets),

(2) $M_{C}$ lies in ker $\psi$, and

(3) the restriction of $\psi$ to $M_{I-C}$ is a near isomorphism of $M_{I-C}$ to $N$.

Proof. Let $D$ be the maximal finite central projection in $M$ and set $\psi(X)=\phi(X)-\phi\left((X D)^{\sharp}\right)$. There exists, by the preceding corollary, a central projection $C \in M$ such that $\phi^{-1}\left(Z_{N}\right)=M_{C}+Z_{M}$. It is easy to see that $\phi^{-1}\left(Z_{N}\right)=\psi^{-1}\left(Z_{N}\right)=T(\operatorname{ker} \psi)$ where $T(U), U$ a Lie ideal, is as above. The definition of $T(\operatorname{ker} \psi)$ implies that $\left[M_{C}, M_{C}\right] \leqq \operatorname{ker} \psi$. By Sunochi's result, $(I-D)\left[M_{C}, M_{C}\right]=M_{C(I-D)} \subseteq$ ker $\psi$. Hence $\psi(X C(I-D))=0$ or $\psi(X C)=\psi(X C D)$. Moreover, again by Sunouchi, $\left[M_{C D}, M_{C D}\right]^{-\|\|}=\left\{X C D \mid(X C D)^{\sharp}=0\right\} \subseteq \operatorname{ker} \psi$. Thus $\psi\left(X C D-(X C D)^{\sharp}\right)=0$. Finally, $\psi(X C)=\psi(X C D)=\psi\left(\left(X C D\left(^{\sharp}\right)=\phi\left((X C D)^{\sharp}\right)-\phi\left((X C D)^{\sharp}\right)=0\right.\right.$.

If $Y \in N$ then, since $\phi$ is $L$-onto, there exists $X \in M$ such that $\phi(X)-Y=Z \in Z_{N}$. Hence $\psi(X)=\phi(X)-\phi\left((X D)^{\sharp}\right)=Y+Z-\phi((X D)$. Since $\phi\left(Z_{M}\right) \subseteq Z_{N}$ we have $\psi(X)-Y \in Z_{N}$.

If $\psi(X(1-C))=\psi(Y(1-C))$ then $\phi(X(1-C)-Y(1-C)) \in Z_{N}$ or $X(1-C)-Y(1-C)=W C+Z$ for $W \in M, Z \in Z_{M}$. Thus $X(1-C)-$ $Y(1-C)=Z(1-C) \in Z_{M}$.

We are now in a position to prove the main result. Note that if $\phi: M \rightarrow N$ is a near isomorphism of von Neumann algebras $M, N$ and if $C_{M}$ (resp $C_{N}$ ) is the maximal central abelian projection in $M$ $(\operatorname{resp} N)$ then $\psi(X)=\left(I-C_{N}\right) \phi\left(X\left(I-C_{M}\right)\right)$ is a near isomorphism of $M_{I-C_{M}}$ to $N_{I-C_{N}}$.

THEOREM 6. If $\phi$ is a uniformly continuous Lie*-homomorphism of a $C^{*}$-algebra $\mathscr{A}$ onto a $C^{*}$-algebra $\mathscr{B}$, there exists a central projection $D$ in the weak closure of $B$ such that (modulo a $\lambda$-map) $D \phi$ is a *-homomorphism and $(1-D) \phi$ is the negative of $a^{*}$-antihomomorphism. 
Proof. Let $M=\mathscr{A}^{* *}$, the second dual of $\mathscr{A}$, and $N=\mathscr{B}^{-W}$. The theorem of Stormer [14: Theorem 3.1, p. 443] for Jordan *-homomorphisms can be altered to give, in the present case, the existence of an $U W$-continuous extension $\tilde{\phi}: M \rightarrow N$ of $\phi$ which is also a Lie *-homomorphism onto.

Let $\psi: M \rightarrow N$ be an $L$-onto Lie *-homomorphism such that there exists a central projection $C$ in $M$ such that $\psi-\tilde{\phi}$ is a $\lambda$-map, $M_{C} \subseteq$ ker $\psi$, and $\left.\psi\right|_{M_{I-C}}$ is a near isomorpism of $M_{I-C}$ to $N$. We can assume, by the above remark, that $M_{I-C}$ and $N$ have no type $I$ summands. Applying Theorem 3 to $\left.\psi\right|_{M_{I-C}}$ we have the desired result.

REMARK 1. Theorem 6 would be an exact analog of Stormer's generalization [13, Theorem 3.3, p. 445] of the Kadison result [6, Theorem $10, \mathrm{p} .334]$ on Jordan homomorphisms of $C^{*}$-algebras were it not for the assumptions of uniform continuity and ontoness. In particular a Jordan *-homomorphism is automatically uniformly continuous. The question of continuity of Lie *-homomorphisms of von Neumann algebras, because of the presence of $\lambda$-maps, is closely connected with the problem of determining the linear span of commutators in rings of operators.

We must assume ontoness in our theorem because of the lack of an analog for the Lie case, to a theorem of Jacobsen and Rickart [16, Theorem 7, p. 487] which states that any Jordan homomorphism of an $m \times n$ matrix ring into another ring is the sum of a homomorphism and an anti-homomorphism.

REMARK 2. Let $\mathscr{A}$ and $\mathscr{B}$ be $C^{*}$-algebras with $I, \mathscr{A} u, \mathscr{B}_{u}$ their respective unitary groups, and $\rho: \mathscr{A}_{u} \rightarrow \mathscr{B}_{u}$ a uniformly continuous group homomorphism. As in [12] there exists uniformly continuous map $\phi: \mathscr{A} \rightarrow \mathscr{B}$, which is in particular a Lie *-homomorphism $\phi$ of $\mathscr{A}$ onto $\mathscr{B}$. Thus the statement of Theorem 6 applies to this $\phi$.

\section{REFERENCES}

1. J. Dixmier, Les Algebres D'Operateurs dans L'Espace Hilbertian, Cahiers Scientifiques, Fas. XXV, Gauthier-Villars, Paris (1957).

2. Les $C^{*}$-algebres et Leurs Reprsentations, Cahiers Scientifiques, Fas. XXIX, Gauthier-Villars, Paris (1964).

3. I. N. Herstein, On the Lie and Jordan rings of a simple associative ring, Ameican

J. Math., 77 (1955), 279-285.

4. - Topics in Ring Theory, The University of Chicago Press, Chicago (1969).

5. L. Hua, $A$ theorem on matrices over a field and its applications, J. Chinese Math. Soc., (N. S.) 1 (1951), 110-163.

6. N. Jacobsen and C. E. Rickart, Jordan homomorphisms of rings, Trans. Amer. Math. Soc., 69 (1950), 479-502. 
7. R. V. Kadison, Isometries of operator algebras, Annals of Math., 54, No. 2 (1951), 325-338.

8. W. S. Martindale 3rd. Lie isomorphisms of primitive rings, Proc. Amer. Math. Soc., 14 (1963), 909-916.

9, — Lie isomorphisms of simple rings, J, London Math. Soc., 44 (1969), 213221.

10. L Lie derivations of primitive rings, Michigan Math. J. 11 (1964), 183-187. 11. C. Robert Miers, Lie isomorphisms of factors, Trans, Amer. Math. Soc., 147, January (1970), 55-63.

12. S. Sakai, On the group isomorphism of unitary groups in $A W^{*}$-algebras, Tohoku Math. J. 7 (1955), 87-95.

13. I. M. Singer, Uniformly continuous representations of Lie groups, Annals of Math., 56 (1952), 242-247.

14. E. St $\phi$ rmer, On the Jordan struture of $C^{*}$-algebras, Trans. Amer. Math. Soc., 120 (1965), 438-447.

15. H. Sunouchi, Infinite Lie Rings, Tohoku Math. J. 8 (1956), 291-307.

Received December 30, 1969 and in revised form April 30, 1970.

UNIVERSITY OF VICTORIA 



\title{
PACIFIC JOURNAL OF MATHEMATICS
}

\author{
EDITORS
}

\author{
H. Samelson \\ Stanford University \\ Stanford, California 94305 \\ C. R. HOBBY \\ University of Washington \\ Seattle, Washington 98105
}

J. DugundJI

Department of Mathematics

University of Southern California

Los Angeles, California 90007

RICHARD ARENS

University of California

Los Angeles, California 90024

\section{ASSOCIATE EDITORS}

E. F. BECKENBACH
B. H. NEUMANN

F. WoLF
K. YosHIDA

\section{SUPPORTING INSTITUTIONS}

\author{
UNIVERSITY OF BRITISH COLUMBIA \\ CALIFORNIA INSTITUTE OF TECHNOLOGY \\ UNIVERSITY OF CALIFORNIA \\ MONTANA STATE UNIVERSITY \\ UNIVERSITY OF NEVADA \\ NEW MEXICO STATE UNIVERSITY \\ OREGON STATE UNIVERSITY \\ UNIVERSITY OF OREGON \\ OSAKA UNIVERSITY \\ UNIVERSITY OF SOUTHERN CALIFORNIA
}

\author{
STANFORD UNIVERSITY \\ UNIVERSITY OF TOKYO \\ UNIVERSITY OF UTAH \\ WASHINGTON STATE UNIVERSITY \\ UNIVERSITY OF WASHINGTON \\ $\stackrel{*}{*} \stackrel{*}{*} \stackrel{*}{*}{ }^{*}{ }^{*}$ MERICAN MATHEMATICAL SOCIETY \\ CHEVRON RESEARCH CORPORATION \\ NAVAL WEAPONS CENTER
}

The Supporting Institutions listed above contribute to the cost of publication of this Journal, but they are not owners or publishers and have no responsibility for its content or policies.

Mathematical papers intended for publication in the Pacific Journal of Mathematics should be in typed form or offset-reproduced, (not dittoed), double spaced with large margins. Underline Greek letters in red, German in green, and script in blue. The first paragraph or two must be capable of being used separately as a synopsis of the entire paper. The editorial "we" must not be used in the synopsis, and items of the bibliography should not be cited there unless absolutely necessary, in which case they must be identified by author and Journal, rather than by item number. Manuscripts, in duplicate if possible, may be sent to any one of the four editors. Please classify according to the scheme of Math. Rev. Index to Vol. 39. All other communications to the editors should be addressed to the managing editor, Richard Arens, University of California, Los Angeles, California, 90024.

50 reprints are provided free for each article; additional copies may be obtained at cost in multiples of 50 .

The Pacific Journal of Mathematics is published monthly. Effective with Volume 16 the price per volume (3 numbers) is $\$ 8.00$; single issues, $\$ 3.00$. Special price for current issues to individual faculty members of supporting institutions and to individual members of the American Mathematical Society: $\$ 4.00$ per volume; single issues $\$ 1.50$. Back numbers are available.

Subscriptions, orders for back numbers, and changes of address should be sent to Pacific Journal of Mathematics, 103 Highland Boulevard, Berkeley, California, 94708.

PUBLISHED BY PACIFIC JOURNAL OF MATHEMATICS, A NON-PROFIT CORPORATION

Printed at Kokusai Bunken Insatsusha (International Academic Printing Co., Ltd.), 7-17, Fujimi 2-chome, Chiyoda-ku, Tokyo, Japan. 


\section{Pacific Journal of Mathematics}

J. T. Borrego, Haskell Cohen and Esmond Ernest Devun, Uniquely

representable semigroups on the two-cell .................. 565

Glen Eugene Bredon, Some examples for the fixed point property ........ 571

William Lee Bynum, Characterizations of uniform convexity .......... 577

Douglas Derry, The convex hulls of the vertices of a polygon of order $n \ldots 583$

Edwin Duda and Jack Warren Smith, Reflexive open mappings .......... 597

Y. K. Feng and M. V. Subba Rao, On the density of $(k, r)$ integers ........ 613

Irving Leonard Glicksberg and Ingemar Wik, Multipliers of quotients of

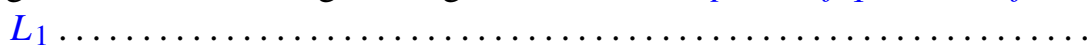

John William Green, Separating certain plane-like spaces by Peano

continua.........................................

Lawrence Albert Harris, A continuous form of Schwarz's lemma in normed

linear spaces .................................... 635

Richard Earl Hodel, Moore spaces and $w$-spaces ............... 641

Lawrence Stanislaus Husch, Jr., Homotopy groups of PL-embedding spaces.

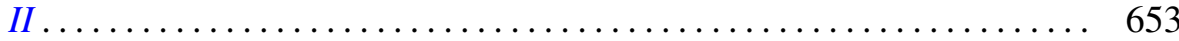

Yoshinori Isomichi, New concepts in the theory of topological

space-supercondensed set, subcondensed set, and condensed set.....

J. E. Kerlin, On algebra actions on a group algebra .................

669

Keizō Kikuchi, Canonical domains and their geometry in $C^{n} \ldots \ldots \ldots \ldots 681$

Ralph David McWilliams, On iterated $w^{*}$-sequential closure of cones. .

697

C. Robert Miers, Lie homomorphisms of operator algebras ..

717

Louise Elizabeth Moser, Elementary surgery along a torus knot ...

737

Hiroshi Onose, Oscillatory properties of solutions of even order differential

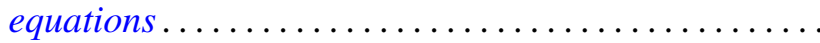

747

Wellington Ham Ow, Wiener's compactification and $\Phi$-bounded harmonic

functions in the classification of harmonic spaces...

Zalman Rubinstein, On the multivalence of a class of meromorphic

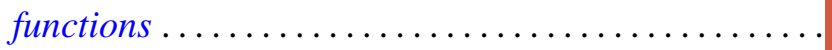

771

785

Hans H. Storrer, Rational extensions of modules

795

topological lattices ...........................

Robert Evert Stong, On the cobordism of pairs .................. 803
Albert Leon Whiteman, An infinite family of skew Hadamard matrices . . . 817

Lynn Roy Williams, Generalized Hausdorff-Young inequalities and mixed

norm spaces 